IC $/ 2001 / 81$

hep-th/0107174

\title{
Brane World in a Topological Black Holes in Asymptotically Flat Spacetime
}

\author{
Donam Youm \\ ICTP, Strada Costiera 11, 34014 Trieste, Italy
}

\begin{abstract}
We study static brane configurations in the bulk background of the topological black holes in asymptotically flat spacetime. We find that such configurations are possible even for flat black hole horizon, unlike the AdS black hole case. We construct the brane world model with an orbifold structure $S^{1} / \mathbf{Z}_{2}$ in such bulk background. We also study massless bulk scalar field.
\end{abstract}

July, 2001

\footnotetext{
${ }^{1}$ E-mail: youmd@ictp.trieste.it
} 
Recently, there has been active interest in the possibility that our four-dimensional universe may be a three-brane embedded in higher-dimensional spacetime 11, 2, 3, 4, as such brane world scenarios might provide possible solutions to the hierarchy and the cosmological constant problems. In the scenario proposed by Randall and Sundrum (RS) [3, 4], the extra spatial dimension needs not be compact for reproducing fourdimensional gravity on the brane due to the strongly warped bulk spacetime and the fine-tuned brane tension is required for solving the hierarchy and the cosmological constant problems.

In particular, the brane universes in the bulk of the five-dimensional AdS black holes [5, 6, 7] and higher-dimensional branes [8] in string theories have been studied. In such models, the motion of the probe brane in the bulk of the source brane is responsible for the expansion of the brane universe on the probe brane. When the equation of state for the matter field on the brane takes a special form, the static brane configuration, in which the probe brane remains static in the bulk, is possible. Such static brane configurations may be regarded as generalizations of the static RS model [3, 4] to different bulk spacetimes. In Refs. [9, 10], it was found out that such static brane configuration is possible even without matter fields on the probe brane for some bulk spacetimes. Such result was generalized to the case of the topological AdS black holes in Ref. [11].

In this paper, we study the static brane configurations in the bulk of the topological black holes in asymptotically flat spacetime. Contrary to the previous belief that black holes in asymptotically flat spacetime should have spherical horizon [12, 13], new class of dilatonic $p$-brane solutions in asymptotically flat spacetime where horizon is the maximally symmetric space with any values of the curvature parameter was constructed [14]. In particular, uncharged 0-brane solution with flat horizon can be transformed to the self-tuning flat domain wall solution [15, 16] when the dilaton coupling parameter takes a special value. We find that the static brane configuration is possible for any horizon geometries, unlike the case of the topological AdS black holes studied in Ref. [11]. We obtain the fine-tuned value of the brane tension and the stabilized location of the probe brane in terms of the parameters of the bulk black hole solution. We also study massless scalar field in such bulk background.

We begin by discussing the new class of dilatonic $p$-brane solutions constructed in Ref. [14]. For the purpose of putting the solutions into more convenient forms, we choose to parameterize the action for the $p$-brane solutions in the following form:

$$
S_{p}=\frac{1}{2 \kappa_{D}^{2}} \int d^{D} x \sqrt{-G}\left[\mathcal{R}-\frac{4}{D-2}(\partial \phi)^{2}-\frac{1}{2 \cdot(p+2) !} e^{2 a_{p} \phi} F_{p+2}^{2}\right],
$$

where $\kappa_{D}^{2}$ is the $D$-dimensional gravitational constant, $\phi$ is the dilaton field, $F_{p+2}$ is the field strength of a $(p+1)$-form potential $A_{p+1}$ and $a_{p}$ is the dilaton coupling parameter. The most general form of the action with arbitrary coefficients in front of the kinetic terms, considered in Ref. [14, is related to this action just through rescaling of $\phi$ and $A_{p+1}$ with constant factors. In terms of such new parametrization, the solution 
for the topological non-extreme dilatonic $p$-brane with the longitudinal coordinates $\mathbf{x}=\left(x^{1}, \ldots, x^{p}\right)$, constructed in Ref. [14, takes the following form:

$$
\begin{aligned}
G_{M N} d x^{M} d x^{N}= & -h_{-}^{\frac{4(n-1)}{(p+n) \Delta_{p}}-1} h_{+} d t^{2}+h_{-}^{\frac{4(n-1)}{(p+n) \Delta_{p}}} d \mathbf{x} \cdot d \mathbf{x}+h_{-}^{-\frac{4(p+1)}{(p+n) \Delta_{p}}-\frac{n-3}{n-1}} h_{+}^{-1} d r^{2} \\
& +h_{-}^{-\frac{4(p+1)}{(p+n) \Delta_{p}}+\frac{2}{n-1}} r^{2} d s_{n, k}^{2}, \\
e^{2 \phi}= & h_{-}^{-\frac{(p+n) a_{p}}{\Delta_{p}}}, \quad A_{t x^{1} \ldots x^{p}}=\frac{2}{\sqrt{\Delta_{p}}} \frac{m \sinh \alpha \cosh \alpha}{r^{n-1}}, \\
h_{+}= & s(r)\left(1-\frac{m \cosh ^{2} \alpha}{r^{n-1}}\right), \quad h_{-}=\left|k-\frac{m \sinh ^{2} \alpha}{r^{n-1}}\right|, \\
\Delta_{p}= & \frac{(p+n) a_{p}^{2}}{2}+\frac{2(p+1)(n-1)}{p+n},
\end{aligned}
$$

where $n \equiv D-p-2, s(r) \equiv \operatorname{sgn}\left(k-m \sinh ^{2} \alpha / r^{n-1}\right)$ and $d s_{n, k}^{2}$ is the metric for the maximally symmetric $n$-dimensional space with constant curvature, which we parametrize as

$$
d s_{n, k}^{2}=\left(1+\frac{k}{4} \delta_{i j} y^{i} y^{j}\right)^{-2}\left[\left(d y^{1}\right)^{2}+\ldots+\left(d y^{n}\right)^{2}\right]
$$

and $k=0, \pm 1$ is the curvature parameter. With our parametrization it is manifest that the curvature singularity at $r=r_{-}=\left(m \sinh ^{2} \alpha\right)^{1 /(n-1)}$ for the $k=1$ case is always covered by a horizon at $r=r_{+}=\left(m \cosh ^{2} \alpha\right)^{1 /(n-1)}$, provided $m>0$. In this paper, we consider the $p=0$ case, for which the solution takes the form:

$$
\begin{aligned}
G_{M N} d x^{M} d x^{N} & =-h_{-}^{\frac{4(n-1)}{n \Delta}-1} h_{+} d t^{2}+h_{-}^{-\frac{4}{n \Delta}-\frac{n-3}{n-1}} h_{+}^{-1} d r^{2}+h_{-}^{-\frac{4}{n \Delta}+\frac{2}{n-1}} r^{2} d s_{n, k}^{2}, \\
e^{2 \phi} & =h_{-}^{-\frac{n a}{\Delta}}, \quad A_{t}=\frac{2}{\sqrt{\Delta}} \frac{m \sinh \alpha \cosh \alpha}{r^{n-1}}, \\
h_{+} & =s(r)\left(1-\frac{m \cosh ^{2} \alpha}{r^{n-1}}\right), \quad h_{-}=\left|k-\frac{m \sinh ^{2} \alpha}{r^{n-1}}\right|, \\
\Delta & =\frac{n a^{2}}{2}+\frac{2(n-1)}{n},
\end{aligned}
$$

where now $D=n+2$ and $a:=a_{0}$ is the dilaton coupling parameter for the $U(1)$ gauge field $A_{M}$. We may regard this topological 0-brane as describing bulk spacetime of a brane world with the worldvolume coordinates $\left(t, y^{1}, \ldots, y^{n}\right)$ and the transverse coordinate $r$. However, unlike the RS model [3, 4, such brane world model generally has asymmetrically warped spacetime, meaning that the space and the time coordinates have different warp factors, and as a result the Lorentz invariance is violated [7]. However, for the uncharged 0-brane solution with $k=0$, the bulk metric can become $(D-1)$-dimensional Poincaré invariant. The uncharged solution in Ref. [14] takes the following form in our parametrization:

$$
G_{M N} d x^{M} d x^{N}=-h_{-}^{\frac{4(n-1)}{n \Delta}-1} h_{+} d t^{2}+h_{-}^{-\frac{4}{n \Delta}-\frac{n-3}{n-1}} h_{+}^{-1} d r^{2}+h_{-}^{-\frac{4}{n \Delta}+\frac{2}{n-1}} r^{2} d s_{n, k}^{2},
$$




$$
e^{2 \phi}=h_{-}^{-\frac{n a}{\Delta}}, \quad h_{+}=\operatorname{sgn} h, \quad h_{-}=|h|, \quad h=k-\frac{l}{r^{n-1}},
$$

where $l$ is an integration constant. Note, this uncharged $p$-brane solution cannot be obtained by taking the zero charge limit of the charged $p$-brane solution (2) ${ }^{2}$. With a choice of $l=-1$ and $k=0$, the metric (5) has the $(D-1)$-dimensional Poincaré invariance, when the temporal and the spatial warp factors have the same $r$-dependence:

$$
r^{-(n-1)\left[\frac{4(n-1)}{n \Delta}-1\right]}=r^{2} \cdot r^{-(n-1)\left[-\frac{4}{n \Delta}+\frac{2}{n-1}\right]} \quad \Longrightarrow \quad \Delta=4 .
$$

For such case, the uncharged solution (5) becomes the self-tuning domain wall solution constructed in Refs. [15, 16], after the redefinition of the transverse coordinate $r$ [14]. So, we see that the self-tuning domain wall solution can be embedded in string theories as an uncharged 0-brane solution with flat $(k=0)$ horizon and $\Delta=4$. Such uncharged 0-brane solution can be obtained by compactifying, for example, uncharged D6-brane, for which $\Delta_{6}=4$, on a compact Ricci flat manifold along its longitudinal directions.

In this paper, we study the brane world on a probe $n$-brane in the bulk background of the topological dilatonic 0-brane (4). For such purpose, it is convenient to redefine the transverse coordinate in the following way [9, 10]:

$$
d z=h_{-}^{-\frac{2}{n \Delta}-\frac{n-3}{n-1}} h_{+}^{-\frac{1}{2}} d r
$$

so that the bulk metric (雨) takes the form that resembles the RS domain wall metric except that the warp factor is asymmetric:

$$
G_{M N} d x^{M} d x^{N}=-A(z)^{2} d t^{2}+B(z)^{2} \sigma_{i j}(x) d x^{i} d x^{j}+d z^{2},
$$

where $A(z)$ and $B(z)$ are implicitly given by

$$
A(z)=h_{-}^{\frac{2(n-1)}{n \Delta}-1} h_{+}^{\frac{1}{2}}, \quad B(z)=h_{-}^{-\frac{2}{n \Delta}+\frac{1}{n-1}} r,
$$

with $r$ related to $z$ in the way defined in Eq. (7). We consider the following form of the $n$-brane action:

$$
S_{\sigma}=-\int d^{n+1} x \sqrt{-g} f(\phi),
$$

where $g$ is the determinant of the induced metric $g_{\mu \nu}=\delta_{\mu}^{M} \delta_{\nu}^{N} G_{M N}$ on the $n$-brane and $f(\phi)$ is an arbitrary function of $\phi$.

We consider the static brane configuration without matter fields on the $n$-brane. From the total action $S_{p=0}+S_{\sigma}$, we obtain the following Einstein's equations and equation of motion for the dilaton field:

$$
\mathcal{G}_{M N}=-\frac{2}{D-2} G_{M N}(\partial \phi)^{2}+\frac{4}{D-2} \partial_{M} \phi \partial_{N} \phi+\frac{1}{2} e^{2 a \phi}\left(F_{M P} F_{N}^{P}-\frac{1}{4} G_{M N} F_{2}^{2}\right)
$$

\footnotetext{
${ }^{2}$ This fact does not imply that our convenient parametrization (2) for the charged topological $p$ brane solution is wrong. This can be easily seen by the fact that the usual uncharged black $p$-brane solution (with $k=1$ ) cannot be coordinate-transformed to take the form (5) with $k=1$.
} 


$$
\begin{gathered}
-\kappa_{D}^{2} \sqrt{\frac{g}{G}} \delta_{M}^{\mu} \delta_{N}^{\nu} g_{\mu \nu} f(\phi) \delta\left(z-z_{b}\right), \\
\frac{4}{D-2} \partial_{M}\left[\sqrt{-G} G^{M N} \partial_{N} \phi\right]=\frac{a}{4} e^{2 a \phi} F_{2}^{2}+\kappa_{D}^{2} \sqrt{-g} f^{\prime}(\phi) \delta\left(z-z_{b}\right),
\end{gathered}
$$

where $\mathcal{G}_{M N}=\mathcal{R}_{M N}-\frac{1}{2} G_{M N} \mathcal{R}$ is the Einstein tensor for the bulk metric $G_{M N}$, the constant $z_{b}$ is the location of the $n$-brane and $D=n+2$. After the explicit expression for the bulk metric (8) is substituted into these equations of motion, the $(z, z)-,(t, t)$ and $(i, j)$-components of the Einstein's equations and the dilaton equation of motion respectively take the following forms:

$$
\begin{gathered}
-\frac{(D-2)(D-3)}{2} \frac{k}{B^{2}}+(D-2) \frac{A^{\prime} B^{\prime}}{A B}+\frac{(D-2)(D-3)}{2} \frac{B^{\prime 2}}{B^{2}} \\
=\frac{2}{D-2}\left(\partial_{z} \phi\right)^{2}-\frac{3}{8} \frac{1}{A^{2}} e^{2 a \phi} F_{t z}^{2}, \\
\frac{(D-2)(D-3)}{2} \frac{k}{B^{2}}-(D-2) \frac{B^{\prime \prime}}{B}-\frac{(D-2)(D-3)}{2} \frac{B^{\prime 2}}{B^{2}} \\
=\frac{2}{D-2}\left(\partial_{z} \phi\right)^{2}+\frac{3}{8} \frac{1}{A^{2}} e^{2 a \phi} F_{t z}^{2}+\kappa_{D}^{2} f(\phi) \delta\left(z-z_{b}\right), \\
\frac{D-3}{2}\left[-(D-4) \frac{k}{B^{2}}+(D-4) \frac{B^{\prime 2}}{B^{2}}+2 \frac{B^{\prime \prime}}{B}+2 \frac{A^{\prime} B^{\prime}}{A B}\right]+\frac{A^{\prime \prime}}{A} \\
\frac{4}{D-2} \partial_{z}\left(A B^{n} \partial_{z} \phi\right)=-\frac{a}{4} \frac{1}{A^{2}} e^{2 a \phi} F_{t z}^{2}+\kappa_{D}^{2} A B^{n} f^{\prime}(\phi) \delta\left(z-z_{b}\right),
\end{gathered}
$$

where the prime denotes the derivative w.r.t. the transverse coordinate $z$.

We now construct the brane world model with an orbifold structure $S^{1} / \mathbf{Z}_{2}$ just like the RS1 model [3]. Following the procedure proposed in Ref. [9], we cut off the transverse space at the black hole horizon $z=z_{H}$ (corresponding to $r=r_{+}=$ $\left.\left(m \cosh ^{2} \alpha\right)^{1 /(n-1)}\right)$ and at the location $z=z_{b}$ of the $n$-brane. The spacetime between these boundaries is referred to as the bulk. We introduce a new transverse coordinate $\bar{z} \equiv z_{b}-z$ and identify the corresponding two points under the $\mathbf{Z}_{2}$-symmetry $\bar{z} \rightarrow-\bar{z}$. So, in this new coordinate the $n$-brane is located at $\bar{z}=0$ and the black hole horizon is located at orbifold points $\bar{z}= \pm\left|z_{b}-z_{H}\right|$, which are identified under the $\mathbf{Z}_{2}$-symmetry $\bar{z} \rightarrow-\bar{z}$. We rewrite the bulk metric (\$) in terms of the new coordinates as

$$
G_{M N} d x^{M} d x^{N}=-A(|\bar{z}|)^{2} d t^{2}+B(|\bar{z}|)^{2} \sigma_{i j}(x) d x^{i} d x^{j}+d \bar{z}^{2}
$$

where $A$ and $B$ are now functions of $|\bar{z}|$ due to the imposed $\mathbf{Z}_{2}$-symmetry and $-\mid z_{b}-$ $z_{H}|\leq \bar{z} \leq| z_{b}-z_{H} \mid$. Although the metric components are continuous at $\bar{z}=0$, their 
first and second derivatives w.r.t. $\bar{z}$ are discontinuous and have $\delta$-function singularity at $\bar{z}=0$, respectively.

By applying the boundary conditions on the first derivatives at $z=z_{b}$ resulting from the $\delta$-function terms in Eqs. (14,15, 16), we can fix the parameters in the $n$-brane action (10) and the (stabilized) location $r_{b}$ of the $n$-brane. For the phenomenological relevance, from now on we consider the $k=0$ case, only \&. By applying the boundary conditions resulting from Eqs. (14,16) we obtain the following constraints on the parameter(s) of $f(\phi)$ :

$$
\begin{gathered}
\kappa_{D}^{2} f\left(\phi_{b}\right)=\frac{4(n-1)}{\Delta} r_{b}^{-1} h_{-}^{\frac{2}{n \Delta}+\frac{n-3}{n-1}}\left(r_{b}\right) h_{+}^{\frac{1}{2}}\left(r_{b}\right), \\
\kappa_{D}^{2} f^{\prime}\left(\phi_{b}\right)=-\frac{4(n-1) a}{\Delta} r_{b}^{-1} h_{-}^{\frac{2}{n \Delta}+\frac{n-3}{n-1}}\left(r_{b}\right) h_{+}^{\frac{1}{2}}\left(r_{b}\right),
\end{gathered}
$$

respectively, where $\phi_{b} \equiv \phi\left(r_{b}\right)$. We note that the following choice of $f(\phi)$ trivially satisfies these constraints:

$$
f(\phi)=\sigma e^{-a \phi},
$$

where $\sigma$ is the $n$-brane tension. The consistency of the boundary conditions resulting from Eqs. (14,15) fixes the stabilized location of the $n$-brane to be

$$
r_{b}^{n-1}=\frac{4-\Delta}{4-2 \Delta} m \cosh ^{2} \alpha .
$$

From this, we see that a real-valued brane position $0 \leq r_{b}<r_{+}$is possible when $0<\Delta<2$ or $\Delta \geq 4$ with $m>0$. This result is in contrast to the case of the uncharged topological AdS black hole, in which the static brane configuration with $k=0$ is possible only when the black hole mass is zero [11. We briefly discuss the limiting cases. When $\Delta=4$, the stable position of the $n$-brane is at $r_{b}=0$, namely at the singularity. When $\Delta=0$, the stable position of the $n$-brane is at the horizon $r_{b}^{n-1}=m \cosh ^{2} \alpha$, meaning that the range of the transverse coordinate $\bar{z}$ is zero, i.e., this case is degenerate. When $\Delta=2$ the static configuration is not possible, since $r_{b}=\infty$ for such case. For the bulk background of the uncharged 0-brane (5) with $k=0$, the consistency of the boundary conditions resulting from Eqs. (14,15) requires that $\Delta=2(n-1)$, meaning that as long as $\Delta=2(n-1)$ the static brane configuration for any values of the $n$-brane location $r_{b}$ is possible. So, the static brane configuration in the background of the self-tuning solution, satisfying (6), is possible only when $n=3$. The following fine-tuned value of the $n$-brane tension is obtained by substituting the

\footnotetext{
${ }^{3}$ The nontrivial static brane configuration is possible also when $k= \pm 1$. The below equations (18) and (19) continue to hold even for the $k= \pm 1$ cases. However, the stabilized brane location $r_{b}$ take more complicated forms $r_{b}^{n-1}=m(2 n-2+n \Delta-2 \Delta) \cosh ^{2} \alpha \sinh ^{2} \alpha /\left[(2 n-2+n \Delta-2 \Delta) \cosh ^{2} \alpha-\right.$ $2 n+2+\Delta]$ and $r_{b}^{n-1}=m(2 n-2+n \Delta-2 \Delta) \cosh ^{2} \alpha \sinh ^{2} \alpha /\left[(2 n-2-n \Delta) \cosh ^{2} \alpha-2 n+2+\Delta\right]$, respectively for $k=1$ and $k=-1$.
} 
expression for $r_{b}$ in Eq. (21) into Eq. (18) along with Eq. (20):

$$
\sigma=\left.\frac{1}{\kappa_{D}^{2}} \frac{4(n-1)}{\Delta} r^{-1} h_{-}^{-\frac{2(\Delta-n+1)}{(n-1) \Delta}} h_{+}^{\frac{1}{2}}\right|_{r=r_{b}}
$$

where $h_{-}\left(r_{b}\right)=\frac{4-2 \Delta}{4-\Delta} \tanh ^{2} \alpha$ and $h_{+}\left(r_{b}\right)=\frac{\Delta}{\Delta-4}$. From this explicit expression for the brane tension, we see that real-valued nonzero brane tension is possible when $\Delta>4$ or $\Delta<0$. So, in order to have the real-valued $n$-brane location and tension, the dilaton coupling parameter $a$ should be such that $\Delta>4$. When $\Delta=4$, for which the $n$-brane is located at the singularity $r=0$, the $n$-brane tension becomes infinite. When $\Delta=0$, for which the $n$-brane is located at the event horizon $r=r_{+}$, the $n$-brane tension is also infinite.

Finally, we study a massless bulk scalar field $\Phi$ satisfying the following equation of motion:

$$
\frac{1}{\sqrt{-G}} \partial_{M}\left(\sqrt{-G} G^{M N} \partial_{N} \Phi\right)=0 .
$$

We take the following ansatz for the solution:

$$
\Phi\left(x^{\mu}, z\right)=u_{m}\left(x^{\mu}\right) f_{m}(z), \quad-A^{-2} \partial_{t}^{2} u_{m}+B^{-2} \delta^{i j} \partial_{i} \partial_{j} u_{m}=-m^{2} u_{m} .
$$

Then, Eq. (23) reduces to the Sturm-Liouville equation:

$$
\partial_{z}\left[A B^{n} \partial_{z}\right] f_{m}=m^{2} A B^{n} f_{m}
$$

In terms of the new $z$-dependent function $\tilde{f}_{m}=A^{\frac{1}{2}} B^{\frac{n}{2}} f_{m}$, this equation takes the following zero energy eigenvalue Schrödinger equation:

$$
-\frac{d^{2} \tilde{f}_{m}}{d z^{2}}+V(z) \tilde{f}_{m}=0
$$

with the potential

$$
V(z)=\frac{1}{2} \frac{A^{\prime \prime}}{A}+\frac{n}{2} \frac{B^{\prime \prime}}{B}+\frac{n}{2} \frac{A^{\prime}}{A} \frac{B^{\prime}}{B}-\frac{1}{4}\left(\frac{A^{\prime}}{A}\right)^{2}+\frac{n(n-2)}{4}\left(\frac{B^{\prime}}{B}\right)^{2}+m^{2} .
$$

This equation can be rewritten in the following convenient form:

$$
\left[\bar{Q} Q+m^{2}\right] \tilde{f}_{m}=0
$$

where

$$
Q=-\frac{d}{d z}+\frac{d \ln A^{\frac{1}{2}} B^{\frac{n}{2}}}{d z}, \quad \bar{Q}=\frac{d}{d z}+\frac{d \ln A^{\frac{1}{2}} B^{\frac{n}{2}}}{d z} .
$$

So, the zero mode $(m=0)$ annihilated by $Q$ is

$$
\tilde{f}_{0}=A^{\frac{1}{2}} B^{\frac{n}{2}}
$$


from which we see that the Kaluza-Klein zero mode $\Phi\left(x^{\mu}, z\right)=u_{0}\left(x^{\mu}\right) f_{0}(z)=$ $u_{0}\left(x^{\mu}\right) A^{-\frac{1}{2}} B^{-\frac{n}{2}} \tilde{f}_{0}$ of the bulk scalar field is independent of $z$, just as in the symmetrically warped brane world case. It is straightforward to show that such result continues to hold for other bosonic fields. In order for the eigenvalue problem for the SturmLiouville equation to be well-defined, appropriate boundary conditions have to be satisfied. For the Sturm-Liouville equation of the form (25), the eigenvalue $m^{2}$ is real and the eigenfunctions $f_{m}$ with different eigenvalues are orthogonal w.r.t. the weighting function $w(z)=A B^{n}$, provided the operator $\mathcal{L}=\partial_{z}\left[A B^{n} \partial_{z}\right]$ is self-adjoint. The operator

$\mathcal{L}$ is self-adjoint, in general when the boundary condition $\left.A B^{n}\left(f_{m}^{\prime} f_{m^{\prime}}-f_{m^{\prime}}^{\prime} f_{m}\right)\right|_{z_{b}} ^{z_{H}}=0$ is satisfied. There are many ways in which this boundary condition can be satisfied. One of possible ways would be to first write the eigenfunction as $f_{n}(z)=F_{m}\left(m^{2} z\right)$ and require that $F_{m}\left(m^{2} z\right)$ vanishes at the boundaries. This requirement determines the eigenvalues $m^{2}$ satisfying the required boundary condition.

\section{References}

[1] N. Arkani-Hamed, S. Dimopoulos and G. Dvali, "The hierarchy problem and new dimensions at a millimeter," Phys. Lett. B429 (1998) 263, hep-ph/9803315.

[2] I. Antoniadis, N. Arkani-Hamed, S. Dimopoulos and G. Dvali, "New dimensions at a millimeter to a Fermi and superstrings at a TeV," Phys. Lett. B436 (1998) 257, hep$\mathrm{ph} / 9804398$.

[3] L. Randall and R. Sundrum, "A large mass hierarchy from a small extra dimension," Phys. Rev. Lett. 83 (1999) 3370, hep-ph/9905221.

[4] L. Randall and R. Sundrum, "An alternative to compactification," Phys. Rev. Lett. 83 (1999) 4690, hep-th/9906064.

[5] P. Kraus, "Dynamics of anti-de Sitter domain walls," JHEP 9912 (1999) 011, hepth/9910149.

[6] D. Ida, "Brane-world cosmology," JHEP 0009 (2000) 014, gr-qc/9912002.

[7] C. Csaki, J. Erlich and C. Grojean, "Gravitational Lorentz violations and adjustment of the cosmological constant in asymmetrically warped spacetimes," Nucl. Phys. B604 (2001) 312, hep-th/0012143.

[8] A. Kehagias and E. Kiritsis, "Mirage cosmology," JHEP 9911 (1999) 022, hepth/9910174.

[9] C. Gomez, B. Janssen and P.J. Silva, "Brane world with bulk horizons," JHEP 0004 (2000) 027, hep-th/0003002.

[10] A. Kamenshchik, U. Moschella and V. Pasquier, "Chaplygin-like gas and branes in black hole bulks," Phys. Lett. B487 (2000) 7, gr-qc/0005011.

[11] D. Birmingham and M. Rinaldi, "Brane world in a topological black hole bulk," hepth/0106237. 
[12] S.W. Hawking, "Black holes in general relativity," Commun. Math. Phys. 25 (1972) 152.

[13] J.L. Friedman, K. Schleich and D.M. Witt, "Topological censorship," Phys. Rev. Lett. 71 (1993) 1486, Erratum-ibid. 75 (1993) 1872, gr-qc/9305017.

[14] C. Grojean, F. Quevedo, G. Tasinato and I. Zavala C., "Branes on charged dilatonic backgrounds: Self-tuning, Lorentz violations and cosmology," hep-th/0106120.

[15] N. Arkani-Hamed, S. Dimopoulos, N. Kaloper and R. Sundrum, "A small cosmological constant from a large extra dimension," Phys. Lett. B480 (2000) 193, hep-th/0001197.

[16] S. Kachru, M. Schulz and E. Silverstein, "Self-tuning flat domain walls in 5d gravity and string theory," Phys. Rev. D62 (2000) 045021, hep-th/0001206. 